\title{
Abertura à Experiência e Preferência por Paisagens Naturais: uma Abordagem Evolucionista
}

\author{
Silvio José Vasconcellos \\ Universidade Federal de Santa Maria \\ Santa Maria, RS, Brasil \\ Cláudio Simon Hutz \\ Universidade Federal do Rio Grande do Sul \\ Porto Alegre, RS, Brasil
}

\begin{abstract}
RESUMO
A psicologia evolucionista concebe a personalidade em termos de estruturas de inferência que se manifestam como tendências ou disposições comportamentais. Nessa perspectiva, o Modelo dos Cinco Grandes Fatores da personalidade referem-se a cinco dimensões amplas influenciadas por sistemas motivacionais. Este artigo apresenta um estudo avaliando a relação entre abertura a experiências e preferências por determinados ambientes naturais. $\mathrm{O}$ estudo também investigou a escolha por ambientes considerados mais seguros pelos participantes. Duas facetas da Escala Fatorial de Abertura a Experiência (Atitudes e Fantasia) mostraram-se positivamente correlacionadas com as medidas de percepção de segurança, mas nenhuma faceta da escala correlacionou-se com as preferências simples. A pesquisa sugere a necessidade de métodos mais acurados para a investigação da relação entre personalidade e a preferência por determinadas condições ambientais.
\end{abstract}

Palavras-chave: Psicologia evolucionista; Psicologia ambiental; Abertura à experiência

\section{ABSTRACT}

\section{Openness to Experience and Preferences for Natural Landscapes: an Evolutionary Approach}

Evolutionary psychology conceptualizes personality in terms of inferred structures that manifest themselves as behavioral tendencies or dispositions. In this perspective the Big Five Personality Model consists of five broad dimensions which are also influenced by motivational systems. The present paper presents a study assessing the relationship between Openness to experience and preferences among natural landscapes. The study also investigated the choice for environments considered safer by the participants. Two facets of the Scale of Openness to Experience were positively related to measures of perception security, but no facet correlated with measures of simple preferences. The research suggests the need for more accurate methods to investigate the relationship between personality and preference for environmental conditions.

Keywords: Evolutionary psychology; Enviromental psychology; Openness to experience

\section{RESUMEN}

\section{Apertura a la Experiencia y la Preferencia por las Paisajes Naturales: una Abordagem Evolucionista}

La Psicología Evolucionista concibe la personalidad en términos de estructuras de inferencia que se manifiestan como disposiciones conductuales o tendencias. En esta perspectiva, el modelo de cinco factores de personalidad se relaciona con cinco grandes dimensiones influenciadas por los sistemas motivacionales. Este trabajo presenta un estudio sobre la relación entre la apertura a la experiencia y las preferencias por ciertos ambientes naturales. El estudio también investigo la elección para entornos considerados más seguros para los participantes. Dos facetas de la Escala Factorial de Apertura a la Experiencia (Actitudes y Fantasía) se correlacionaron positivamente con las medidas de seguridad que se percibe pero ninguna faceta se correlacionó con las preferencias simples. La investigación sugiere la necesidad de métodos más precisos para investigar la relación entre la personalidad y la preferencia por ciertas condiciones ambientales.

Palabras clave: Psicología evolucionista; Psicología ambiental; Apertura a la experiencia 
O termo adaptação refere-se a qualquer característica ou comportamento natural evoluido que torna algum organismo capacitado a sobreviver em seu respectivo habitat. $\mathrm{O}$ ser humano evoluiu expandindo inúmeras habilidades que o permitiram ocupar diferentes tipos de habitats (Foley, 1998). Nesse sentido, não é possível pensar em locais e condições ambientais que possam, de forma exclusiva, terem favorecido a evolução dos hominídeos.

Mostra-se possível, de outra forma, compreender que alguns tipos de ambientes podem reunir condições adaptativas mais proeminentes. Appleton (1975) foi quem pela primeira vez postulou que um juízo estético sobre certo ambiente poderia estar vinculado a uma função adaptativa em termos de espécie. Sua acepção é de que os seres humanos preferem paisagens que evoquem, conjuntamente, uma condição de perspectiva e refúgio. Segundo esse autor, o termo perspectiva remete-nos à possibilidade de ter uma ampla visão da paisagem, enquanto que o termo refúgio refere-se à possibilidade de ter um local para se esconder, onde seja possível enxergar sem ser enxergado (Appleton, 1975). Balling e Falk (1982) evidenciaram, de outro modo, dados condizentes com a assim denominada hipótese da Savana. Ou seja, ao serem avaliadas as preferências diante dos cinco tipos de vegetação mais presentes no mundo, os indivíduos tendiam a preferir savanas, ainda que nunca tivessem estado em uma. Num estudo transcultural, Herzog, Herbert, Kaplan e Crooks (2000) evidenciaram uma preferência por cenários onde rios estivessem presentes. Kaplan, Kaplan e Brown (1989) enfatizam ainda que o próprio grau de mistério que caracteriza diferentes cenários pode apresentar-se como um "elemento chave", capaz de influenciar preferências e avaliações estéticas.

Mealey e Theis (1995), ao compararem a preferência por cenas ricas em perspectivas com cenas ricas em refúgio numa amostra de 50 indivíduos, constataram que tensão, ansiedade e fadiga mostraram-se associadas à preferência por ambientes de refúgio. Em um estudo complementar feito com 80 indivíduos, Klopp e Mealey (1998) encontraram dados discordantes do trabalho de Mealey e Theis (1995), sendo que a indução quanto ao estado de humor dos participantes não se mostrou preditiva para a escolha de ambientes específicos.

Quando é comparada e discutida a preferência por ambientes que oferecem maior possibilidade de refúgio ou maior possibilidade de perspectiva, em cenários naturais ou urbanos, observa-se que não há uma completa convergência de resultados. De um lado, pesquisas que evidenciam a influência da perspectiva e refúgio nas escolhas, bem como um estudo que sugere que a capacidade de oferecer refúgio pode não ser determinante nesse processo (Mumcu, Duzenli, \& Ozbilen, 2010). Observa-se, no entanto, uma maior convergência em trabalhos sobre o tema, quanto à noção de que uma busca por refúgio, capaz de diminuir o comportamento exploratório, é uma característica dos seres humanos, bem como de outras espécies em situações de maior ameaça (Ramanujam, 2006).

De acordo com Kaplan (1992), o estudo da preferência por determinados ambientes naturais constituise uma das formas promissoras para estudar o impacto da evolução sobre as tendências comportamentais humanas. Autores como Bouchard e Loehlin (2001) assinalam que a evolução encarregou-se de selecionar repertórios comportamentais que constituem os diferentes aspectos da personalidade. Considerando que tais aspectos podem, por certo, ser constitutivos da perssoanlidade, Buss (1999), de forma mais peremptória, afirmou que "teorias da personalidade inconsistentes com princípios evolutivos têm poucas ou nenhuma chance de estarem corretas" (p. 52).

Neste artigo, investigou-se aspectos da personalidade relacionados à preferência por determinados tipos de ambientes naturais. A hipótese central refere-se a uma possível relação entre aspectos da personalidade considerados, dessa forma, como tendências comportamentais específicas e a preferência relacionada a uma maior sensação de segurança. Os aspectos investigados são facetas de um dos cinco grandes fatores constituintes da personalidade, denominado abertura à experiência. Tais fatores são dimensões amplas da personalidade reveladas a partir de estudos que empregam o método da análise fatorial, objetivando identificar o modo com as características da personalidade humana podem ser agrupadas. Esse modelo tem recebido um considerável suporte empírico a partir de inúmeros estudos transculturais desenvolvidos nas últimas décadas. Os demais fatores contemplados no modelo denominam-se: extroversão, socialização, realização e neuroticismo (Hutz et al., 1998).

O fator abertura à experiência abarca uma série de comportamentos exploratórios e envolvimento com novas experiências. Indivíduos com pontuações altas em escalas que avaliam o fator mostram-se propensos a manifestar uma maior curiosidade, imaginação, criatividade, sendo que pontuações altas também podem evidenciar uma tendência para valorizar ideias e padrões de comportamentos não convencionais (Costa \& Widiger, 1993).

Explicações evolucionistas têm sido vinculadas ao modelo dos cinco grandes fatores. Conforme explica Golberg (1981), as pessoas costumam fazer algumas perguntas básicas e universais quando interagem com 
outras pessoas: $\mathrm{X}$ é ativo e dominador ou passivo e submisso (Posso mandar em $\mathrm{X}$ ou $\mathrm{X}$ tentará mandar em mim)?; X é agradável (afetuoso e amável) ou desagradável (frio e distante)?; Posso contar com X (X é responsável e consciente ou é omisso e negligente)?; X é louco (imprevisível) ou é (estável)?; e X é esperto ou estúpido?; Tem interesse naquilo que não diz respeito a sua experiência de vida cotidiana? (será fácil para mim ensinar algo a $\mathrm{X})$ ?

Para MacDonald (1995), os grandes fatores da personalidade reúnem funções adaptativas cruciais para a sobrevivência. Para o autor, o fator abertura à experiência atrela-se à necessidade de explorar o próprio ambiente em busca de novos recursos. Na sequência descreve-se o estudo que investigou a relação entre abertura à experiência com a preferência por ambientes naturais (de perspectiva e refúgio). Além dos métodos já empregados em outros trabalhos (Mealey \& Theis, 1995; Klopp \& Mealey, 1998), envolvendo a escolha simples na qual o participante apenas indicou as fotos que retratam ambientes de sua preferência, também foi investigada a relação entre as facetas desse fator com o número de vezes que os participantes escolheram fotos de perspectiva como retratando os ambientes nos quais se sentiam mais seguros. Dentro dessa abordagem, faz-se necessário ressaltar ainda que ambientes heterogêneos podem favorecer tendências evolutivas semelhantes (Holt \& Gaines, 1992). Ambientes que oferecem mais perspectiva e menos refúgio, conjuntamente com ambientes que oferecem mais refúgio poderiam favorecer a preferência destacada por Appleton (1975), não havendo, portanto um ambiente evolutivo invariável no que se refere a essas mesmas características.

\section{MÉTODO}

\section{Participantes}

Um total de 209 indivíduos (116 mulheres e 93 homens) estudantes do ensino superior, pertencentes a uma universidade particular e uma universidade pública, participou desse estudo. A idade dos participantes variou de 17 até 56 anos $(M=23,7$ e $\mathrm{DP}=7,4)$. A amostra contou com estudantes de diferentes cursos, tais como Psicologia, Engenharia Civil, Sociologia. Educação Física e outros, mas excluiu estudantes dos cursos de Arquitetura e Engenharia Florestal, considerando uma possível tendência que os estudantes e profissionais com tal formação poderiam apresentar no que se refere a atentar para a funcionalidade do ambiente a partir de outros aspectos. A exclusão de estudantes e profissionais de Arquitetura ou áreas afins mostrou-se apropriada em estudos anteriores.

\section{Material}

Foram apresentadas 10 fotos coloridas dispostas em uma única folha, das quais cinco expressavam uma condição de perspectiva e outras cinco expressavam uma condição de refúgio Cada foto constante na folha media $8 \mathrm{~cm}$ X 5,5 cm. A seleção das fotos utilizadas contou com a participação de 150 voluntários (estudantes e profissionais de diferentes áreas) que avaliaram 20 fotos selecionadas pelos pesquisadores, identificando ambientes que ofereciam uma condição de perspectiva ou uma condição de refúgio, conforme as definições estabelecidas por Appleton (1975) e que foram previamente fornecidas para os avaliadores. Cada uma das fotos selecionadas foi considerada como expressando uma condição exclusivamente de refúgio ou exclusivamente de perspectiva para, no mínimo, 95\% da amostra de voluntários participantes dessa etapa.

Todas as fotos que compuseram o estudo foram de ambientes naturais nos quais não havia qualquer sinal de intervenção humana deliberada e onde nenhum tipo de fonte de água (rio, cachoeira, lago) estivesse presente. Todas as fotos foram tiradas no mesmo dia e em horários próximos, nos quais não foi possível identificar nenhum tipo de variação quanto ao grau de luminosidade.

Para a avaliação do fator de personalidade Abertura dos participantes foi utilizada a Escala Fatorial de Abertura à Experiência (EFA) constituída como um inventário de autorrelato com 42 itens e mensura as facetas: atitudes, hábitos e valores e fantasia. A primeira dessas facetas comporta 16 itens e relaciona-se a uma série de atitudes que envolvem a experimentação da mudança, a tendência para não estabelecer rotinas, bem como explorar ambientes e situações novas. A segunda faceta comporta 17 itens e volta-se para uma série de valores morais e a consequência tendência do indivíduo repensar e refletir sobre esses mesmos valores. A terceira faceta abarca 9 itens e contempla o pensamento imaginativo e a capacidade do indivíduo vislumbrar situações inusitadas. Os itens de cada faceta são respondidos utilizando uma escala Likert de 7 pontos. A escala foi construída e validada por Vasconcelos (2007) e Vasconcellos e Hutz (2008), sendo utilizada tão somente para avaliar um dos cinco grandes fatores da personalidade.

\section{Procedimentos}

A pesquisa realizada obteve aprovação do Comitê de Ética em Pesquisa da Universidade Federal do Rio Grande do Sul. Todos os participantes foram avaliados de forma coletiva em salas de aulas em um 
tempo aproximado de 35 minutos. Todos receberam informações quanto ao fato de que a participação seria voluntária, podendo, os mesmos, recusarem-se a participar. Após a assinatura do termo de consentimento livre e esclarecido, houve o preenchimento da EFA, cada participante era solicitado a olhar uma folha na qual as fotos estavam contidas, sendo que as mesmas foram apresentadas sempre na mesma ordem para todos os participantes. Na sequência, os participantes assinalaram, em uma grade de respostas, cinco fotos expressando apenas a sua preferência pelas mesmas. Após assinalarem suas fotos preferidas, os participantes foram solicitados a escolher, assinalando em outra grade de respostas, as fotos que, conforme seu entendimento, retratavam os ambiente nos quais se sentiam mais seguros.

\section{RESULTADOS}

$\mathrm{Na}$ Tabela 1 são apresentadas as correlações (Correlação de Spearman) obtidas entre o número de fotos de perspectiva escolhidas nas duas condições e as diferentes subescalas da EFA, bem como escore total nessa mesma escala. Optou-se por uma análise das escolhas realizadas nas duas etapas apenas em termos de variáveis contínuas, pois não era objetivo do presente trabalho examinar todas as variáveis que pudessem influenciar na escolha de cenários específicos retratados em cada uma das fotos isoladamente. Em termos gerais, os dados obtidos indicam existir correlação estatisticamente significativa com duas facetas da escala, bem como escore total quando os participantes foram solicitados a indicar o local no qual se sentiam mais seguros.

\section{TABELA 1}

Subescalas EFA, preferência e atribuição de segurança para ambientes que expressam condição de perspectiva

\begin{tabular}{lccc}
\hline Subescala & Média & $\begin{array}{c}\text { Correlação com } \\
\text { preferências por } \\
\text { ambientes de } \\
\text { perspectiva }\end{array}$ & $\begin{array}{c}\text { Correlação com } \\
\text { atribuição de } \\
\text { segurança para } \\
\text { ambientes de } \\
\text { perspectiva }\end{array}$ \\
\hline Hábitos e valores & 70,72 & $-0,02$ & $-0,11$ \\
Atitude & 78,69 & $-0,04$ & $0,20^{*}$ \\
Fantasia & 28,18 & 0,02 & $0,26^{*}$ \\
Escore total & 69,02 & $-0,03$ & $0,16^{*}$ \\
\hline
\end{tabular}

$* p<.001$.

O teste t de Student, conjuntamente com o tamanho do efeito calculado como forma de avaliar a magnitude das diferenças encontradas, indicaram a existência de diferenças estatisticamente significativas nas duas situações conforme evidencia a Tabela 2.
TABELA 2

Comparação entre as duas condições de escolha das fotos de perspectiva para o mesmo grupo

\begin{tabular}{lcccccc}
\hline $\begin{array}{c}\text { Escolha } \\
\text { envolvendo } \\
\text { preferência } \\
\text { simples }\end{array}$ & $\begin{array}{c}\text { Escolha } \\
\text { envolvendo } \\
\text { atribuição de } \\
\text { segurança }\end{array}$ & Significancia & $\begin{array}{c}\text { Tamanho } \\
\text { do Efeito }\end{array}$ \\
\hline Média & $D P^{*}$ & Média & $D P^{*}$ & $t$ & $p$ & $d$ \\
\hline 2.11 & 1.52 & 0,69 & 1.59 & 23,25 & .001 & .91 \\
\hline * Desvio-Padrão. & & & & &
\end{tabular}

\section{DISCUSSÃO}

De um modo diferente dos trabalhos de Mealey e Theis (1995), o estudo apresentado contou com a classificação de ambientes de perspectiva e ambientes de refúgio feita por um grupo de 150 voluntários e não apenas pelos pesquisadores. Por outro lado, houve concordância com o citado trabalho no que se refere a uma aproximação entre a média de escolhas de ambiente de perspectiva e a média de escolhas de ambiente de refúgio na amostra total. Conforme salientou Appleton (1975), os ambientes considerados ideais abarcam tanto perspectiva como refúgio. $\mathrm{Na}$ pesquisa em questão, trabalhou-se com ambientes que expressam exclusivamente a condição de perspectiva ou exclusivamente a condição de refúgio.

Observa-se, em contrapartida, que os resultados obtidos neste trabalho sugerem que o processo simples de escolha pode não assinalar uma preferência baseada no tipo de ambiente que os indivíduos consideram mais seguros. Em trabalho anterior (Mealey \& Theis, 1995), aventou-se que tendências específicas relacionadas à escolha das fotos poderiam estar fundamentadas em mecanismos adaptativos, considerando que alguns ambientes podem ter oferecido maiores condições de segurança ao longo da nossa história filogenética. Nesses termos, o ambiente preferencial seria, conforme Appleton (1975), aquele que oferece, ao mesmo tempo, condições de perspectiva e refúgio. Quando há uma escolha forçada entre ambientes que oferecem exclusivamente uma ou outra condição, de forma dicotômica, torna-se então possível investigar as características que prevalecem no processo de escolha. Tais escolhas poderiam, conforme esse entendimento, estar correlacionadas a níveis de ansiedade, induzida ou não, porém sugestivos quanto ao estado motivacional do indivíduo, capaz de levá-lo a preferir um ou outro tipo de ambiente. Ratifica-se, porém, que esse mesmo processo de escolha pode não estar sendo necessariamente determinado por uma maior ou menor atribuição de segurança quanto ao tipo de ambiente, conforme sugere o presente trabalho. 
Faz-se necessário considerar a relação do fator abertura à experiência com o processo de escolhas das fotos que retratavam ambientes de perspectiva ou ambientes de refúgio, tendo sido essa a investigação basilar do estudo proposto. Conforme ilustra a tabela 1, não houve correlação estatisticamente significativa entre as facetas da EFA, bem como entre o escore total da escala com a escolha dos indivíduos, quando os mesmos foram solicitados tão somente a assinalar fotos de sua preferência. Por outro lado, algumas correlações positivas e estatisticamente significativas, ainda que baixas, foram obtidas com as facetas atitude e fantasia quando o processo de escolha pautou-se pela possibilidade do ambiente oferecer um maior sentimento de segurança. Não houve correlação estatisticamente significativa entre a faceta hábitos e valores com essa mesma variável.

Os resultados sugerem, portanto, que indivíduos com escores mais altos na faceta atitude e na faceta fantasia estariam um pouco mais propensos a conceberem ambientes de perspectiva como sendo mais capazes de oferecer segurança. Em outras palavras, tais características da personalidade podem, conforme indicam os índices de correlação obtidos, também influenciar em uma maior ou menor atribuição de segurança ao ambiente, ainda que não tenham o mesmo grau de influência no processo de escolha simples.

Considerando-se, por exemplo, que a faceta atitude envolve a descrição de uma série de comportamentos nos quais os indivíduos podem se deparar com aspectos não previstos de uma situação, aventa-se que indivíduos com escores mais altos nessa faceta poderiam não se sentir tão expostos ao risco em ambientes abertos, conforme tende a ocorrer na amostra total. Há uma tendência geral para uma maior atribuição de segurança em ambientes de refúgio, considerando que a os níveis de escolha de ambientes de perspectiva como sendo aqueles que oferecem mais segurança foi significativamente menor na segunda etapa. Considerase, de outro modo, que essa mesma atribuição pode ser menos preponderante em indivíduos com um maior nível de abertura à experiência, ao menos no que se refere a determinados aspectos desse construto.

Em uma perspectiva evolucionista, constata-se que teorias, a exemplo da denominada Teoria do Prospecto (Zucherman, Eysenck, \& Eysenck, 1978; Arnett, 1994), postulam que o risco é inerente a uma alternativa com base na variância da recompensa. Entretanto, em relação ao que se pode entender por recompensa, outras abordagens concebem-na como sendo dependente de características da personalidade, incluindo uma maior ou menor tendência pela busca de novas sensações (Zucherman, Eysenck, \& Eysenck, 1978; Arnett, 1994). Uma teoria desse tipo objetiva examinar a propensão ao risco nos seres humanos e as tendências comportamentais que a ela estão relacionadas. Nesses termos, ambientes de perspectiva oferecem, nesse sentido, uma maior estimulação sensorial e, conforme características específicas da personalidade relacionadas à atitude e à imaginação, esse maior nível de estimulação poderia então afetar a atribuição de risco para o cenário vislumbrado. Contribuiriam, portanto, para que o indivíduo tenha a tendência a percebê-lo como oferecendo menos riscos.

De um modo geral, faz-se necessário uma investigação mais criteriosa envolvendo tais considerações como forma de gerar achados mais consistentes sobre a influência de aspectos da personalidade no que se refere à escolha por determinados tipos de ambientes, bem como à propensão ao risco. O presente trabalho valeu-se de uma amostra pequena, porém suficiente para gerar achados sugestivos quanto a uma possível relação entre traços da personalidade e escolha por ambientes naturais. Além disso, problematizou aspectos metodológicos que fundamentaram alguns estudos anteriormente desenvolvidos sobre a relação entre estados de humor e escolha por ambientes naturais. Constata-se a necessidade de novos trabalhos nessa área, com o intuito de investigar melhor a relação entre abertura à experiência, ou mesmo outros fatores da personalidade com a tendência para avaliar as características de ambientes naturais ou urbanos. Uma investigação mais criteriosa nesse campo pode fomentar avanços no campo da Psicologia Ambiental, bem como da própria Psicologia Evolucionista, buscando a integração desse campo com diferentes abordagens teóricas. Para que algumas hipóteses na esfera da PE, nessa mesma área do conhecimento, possam ser devidamente testadas, faz-se necessário um refinamento de aspectos metodológicos conforme evidenciou o estudo apresentado. Sugerem-se estudos com amostras maiores, bem como o exame de outras variáveis que possam estar relacionadas ao processo de escolha dos ambientes naturais.

\section{REFERÊNCIAS}

Appleton, J. (1975). The experience of landscape. London: Wiley \& Sons.

Arnett, J. (1994). Sensation seeking: A new conceptualization and a new scale. Personality and Individual Differences, 16 , 289-296.

Balling, J. D. \& Falk, J. H. (1982). Development of visual preference for natural environments. Environment and Behavior, 14, $5-28$. 
Buss, D. M. (1999). Human nature and individual differences; the evolution of human personality. In L. A. Pervin \& O. P. John (Orgs.). Handbook of personality: Theory and Research (pp. 31-56). New York: Guilford.

Costa, P. T. \& Widiger, T. A. (1993). Introduction. In P. T. Costa \& T. A. Widiger (Orgs.). Personality Disorders and the five-factor model of personality (pp. 1-10). Washington, DC: American Psychological Association.

Crick, F. (1990). A hipótese espantosa: busca científica da alma. Lisboa: Instituto Piaget.

Foley, R. (2003). Os humanos antes da humanidade: uma perspectiva evolucionista. São Paulo: Editora Unesp.

Golberg, L. (1981). Language and individual differences: the search for universal in personality lexicons. In L. Wheeler (Org.). Review of Personality and social Psychology (pp. 141-165). Beverly Hills: Sage.

Herzog, T. R., Herbert, E. J., Kaplan, R., \& Crooks, C. L. (2000). Cultural and developmental comparisons of landscape perceptions and preferences. Environment and Behavior, 32, 323-346.

Holt, R. D. \& Gaines, M. S. (1992). Analysis of adaptation in heterogeneous landscapes: implications for the evolution of fundamental niches. Evolutionary Ecology, 6, 433-447.

Hutz, C. S., Silveira, A. D., Serra, J. G., Anton, M. C., \& Wieczorek, L. S. (1998). O desenvolvimento de marcadores para a avaliação da personalidade no Modelo dos Cinco Grandes Fatores. Psicologia: Reflexão e Crítica, 11, 395-415.

Kaplan, S. (1992). Environmental preference in a knowledgeseeking, knowledge-using organism. In J. H. Cosmides \& J. Tooby (Orgs.). The Adapted Mind (pp. 581-598). New York: Oxford UP.

Kaplan, R., Kaplan, S., \& Brown, T. (1989). Enviromental Preference. A comparasion of four Domais of predictors. Enviroment and Behavior, 21, 509-530.

Klopp, B. \& Mealey, L. (1998). Experimental Manipulation of Mood Does Not Induce Change in Preferences for Natural Landscapes. Human Nature, 4, 391-399.

MacDonald, K. (1995). Evolution, the five-factor model, and levels of personality. Journal of Personality, 63, 525-567.
Mealey, L. \& Theis, P. (1995). The relationship between mood and preferences among natural landscapes: An evolutionary perspective. Ethology and Sociobiology, 16, 247-256.

Mucmu, S., Duzenli, T., \& Özbilen, A. (2010). Prospect and Refuge as the predictors of preferences for seating areas. Scientific Research and Essays, 5, 1223-1233

Ramanujan, P. (2006). Prospect-Refuge Theory Revisited: A Search for Safety in Dynamic Public Spaces With Reference to Design. Unpublished Thesis. The University of Texas, Arlington.

Reitman, J. S. (1971). Mechanisms of forgetting in shor-term memory. Cognitive Psychology, 2, 185-195.

Sternberg, R. (2000). Psicologia Cognitiva. Porto Alegre: Artmed.

Vasconcellos, S. J. L. (2007). Pressupostos evolucionistas dos fatores neuroticismo e abertura a novas experiências no modelo dos cinco grandes fatores. Tese de Doutorado em Psicologia, Universidade Federal do Rio Grande do Sul. www.lume.ufrgs. br/handle/10183/14918

Vasconcellos, S. J. L. \& Hutz, C. S. (2008). Construção e validação de uma escala de abertura à experiência. Avaliação Psicológica, 7, 135-141.

Zucherman, M., Eysenck, S. B. G., \& Eysenck, H. J. (1978). Sensation seeking in England and America: cross-cultural, age, and sex comparisons. Journal of Consulting and Clinical Psychology, 46, 139-149.

Autores:

Silvio José Lemos Vasconcellos - Doutor, Professor Adjunto do Curso de Psicologia da Universidade Federal de Santa Maria (UFSM).

Cláudio Simon Hutz - Doutor, Professor do Programa de Pós-Graduação em Psicologia da Universidade Federal do Rio Grande do Sul (UFRGS).

Endereço para correspondência:

Silvio José Lemos Vasconcellos

Rua Marechal Floriano Peixoto, 1750, $3^{\circ}$ andar, sala 313

Prédio de Apoio da UFSM - Centro

CEP 97.015-372 Santa Maria, RS, Brasil

E-mail: sjlvasconcellos@bol.com.br

Recebido em: 05.11.12

Aceito em: 14.05.13 